\title{
Apoyo motivacional docente y necesidades psicológicas básicas en la Educación Física: una revisión sistemática
}

Teacher motivational support and basic psychological needs in Physical Education: a systematic review

Julio Alfonso Mocha Bonilla. ${ }^{1}$, Mariuska Comas Benítez. ${ }^{2}, \&$ Eduardo Lautaro Galak. ${ }^{3}$

Recibido: 15-01-2020 / Revisado: 23-01-2020 /Aceptado: 26-01-2020/ Publicado: 27-02-2020

\begin{abstract}
DOI: https://doi.org/10.33262/cienciadigital.v4i1.1.1160

This article establishes a framework of reference on teacher motivation and the significance of support for basic psychological needs in physical education. Dial net and Google academic; using as keywords the terms: "Motivation, BPNES, Physical Education, Teacher Support", in Spanish, English and Portuguese. The period of time considered was the last eleven years. The search carried out allowed to identify several related works, but only the most significant were included in the review. The selection of works included articles, research reports, and book chapters. The analysis of the identified contents was carried out from a perspective of reflection, using analytical-synthetic and inductive-deductive methods. After the analysis of the information gathered, it was possible to conclude that when the teacher support towards the basic psychological needs is provided, a self-determined motivation can be perceived in the educational field of physical education and sport, the integral formation of the students should promote the values through the teacher motivational support, emphasizing the pedagogical aspect in the construction of cognitive and affective values.
\end{abstract}

Keywords: Motivation, BPN-PE, Physical Education, Teacher Support.

\footnotetext{
${ }^{1}$ Universidad Técnica de Ambato, Facultad de Ciencias Humanas y de la Educación, Carrera de Pedagogía de la Actividad Física y Deporte, Ambato, Ecuador, Doctorando en Ciencias de la Educación (UNLP Argentina) ja.mocha@uta.edu.ec

${ }^{2}$ Universidad Técnica de Ambato, Facultad de Ciencias Humanas y de la Educación, Carrera de Educación Básica, Ambato, Ecuador, m.comas@uta.edu.ec

${ }^{3}$ CONICET-Universidad Nacional de la Plata, Facultad de Humanidades y Ciencias de la Educación, La Plata, Argentina, egalak@fahce.unlp.edu.ar
} 


\section{Resumen}

Este artículo establece un marco de referencia sobre la motivacional docente y la significancia del apoyo hacia las necesidades psicológicas básicas en la educación física, Se llevó a cabo una revisión literaria de investigaciones recientes alrededor de la temática objeto de estudio, se utilizaron como fuentes de consulta las bases de datos: Scielo, Redalyc. Dialnet y Google Académico; usando como palabras clave los términos: «Motivación, BPN-PE, Educación Física, Apoyo Docente», en idioma español, inglés y portugués. El período de tiempo considerado fue de los últimos once años. La búsqueda realizada permitió identificar variados trabajos relacionados, pero solamente los más significativos fueron incluidos en la revisión. La selección de trabajos incluyó artículos, informes de investigación, y capítulos de libro. El análisis de los contenidos identificados se realizó en función de una perspectiva de reflexión, empleando los métodos analíticos-sintéticos e inductivos-deductivos. Tras el análisis de la información recopilada, fue posible concluir que cuando se brinda el apoyo docente hacia las necesidades psicológicas básicas se puede percibir una motivación autodeterminada en el ámbito educativo de la educación física y el deporte; la formación integral de los estudiantes debe fomentar los valores a través del apoyo motivacional docente, destacando el aspecto pedagógico en la construcción de valores cognitivos y afectivos.

Palabras claves: Motivación, BPN-PE, Educación Física, Apoyo Docente.

\section{Introducción}

Para el docente de Educación Física (EF) constituye un reto constante lograr la participación activa de los estudiantes en las clases. El rol docente es decisivo en la adquisición de los hábitos educativos, es así como, día a día se buscan las mejores herramientas para generar experiencias positivas y consoliden las mejores condiciones para el aprendizaje (Aelterman, et al 2013). Esto ha sido una preocupación constante de los maestros de Educación Física, el poder incorporar en el proceso de enseñanza-aprendizaje, los conocimientos de motivación docente para la enseñanza del currículo escolarizado, pues el docente especialista: conoce y domina el contenido educativo como parte fundamental de la actividad pedagógica, la relación dialéctica de los objetivos con los contenidos, los 
métodos y medios para alcanzar los resultados planificados. Sin embargo, el feedback ${ }^{4}$ que establezca cada maestro durante la organización de su clase, debe afianzar las condiciones del aprendizaje enfocado mediante la motivación y la práctica motriz en la Educación Física, pues el ejercicio físico actúa en la prevención de las denominadas enfermedades no trasmisibles (Organización Mundial de la Salud, 2016), además, ayuda para que algunos aspectos como el sedentarismo, la obesidad y el tabaquismo se reduzcan o eliminen mediante la práctica de ejercicios (Paiderex, 2011). Por lo tanto, el desarrollo de los programas de Educación Física y deportes en las escuelas elementales y secundarias deben estar enfocadas hacia las actividades psicomotrices y afectivas, incluir conferencias regulares sobre higiene (...) desarrollo de juegos Inter escolares y la necesidad de organizaciones para regular el currículo de Educación Física (Velázquez, 2011) de acuerdo con la realidad socioeducativa.

Dentro del proceso educativo debe existir el par dialéctico pedagógico, donde el docente es el guía y orientador del proceso, mientras que el alumno es el protagonista del aprendizaje. En las clases de Educación Física la gran mayoría son clases prácticas, por consiguiente, la presencia y motivación del docente es necesaria durante el proceso de enseñanza. Al hablar de la práctica y formación del profesorado en la Educación Física, Aranda (2013) sugiere "dar paso al diseño de un currículo abierto, que ayude hacia la construcción de los aprendizajes, donde la cooperación activa de los docentes amerita su desarrollo profesional". La participación activa debe ser la guía mediante procesos de motivación, tanto de los docentes como en los estudiantes durante las actividades físicoescolares, las cuales son muy importantes en el proceso de enseñanza aprendizaje, es decir, los estudiantes perciben la motivación desplegada por cada maestro en sus clases, ya que gracias a ello tendrán la oportunidad de transformar su conducta (Gutiérrez, 2016).

Por lo expuesto, el presente trabajo aborda el estudio de la motivación docente, un contexto en el cual, con el inicio de la teoría de la autodeterminación a nivel mundial, se han realizado y afianzado los estudios sobre el tema del apoyo motivacional docente durante las clases de Educación Física. De esta manera se considera pertinente indagar la Percepción del Apoyo Docente hacia las Necesidades Psicológicas Básicas (NPB) en los estudiantes

\footnotetext{
${ }^{4}$ Feedback: Instancia de retroalimentación o respuesta durante un proceso de comunicación.
} 
escolarizados, lo cual constituye la base sobre la cual se analiza la perspectiva teórica y los fundamentos de apoyo científico.

Por consiguiente, el tema propuesto pretende analizar las prácticas escolarizadas presentando una fundamentación científica mediante la cual se indaga la motivación a través del apoyo de las necesidades psicológicas básicas en los procesos educativos de la Educación Física.

\section{Metodología}

Se realizó una revisión bibliográfica y documental de los artículos científicos publicados desde el periodo comprendido entre el 2009 hasta el mes de febrero del 2020, destacando los más citados.

Para las búsquedas se usaron las siguientes palabras claves: Motivación, BPN-PE, Educación Física, Apoyo docente; en idioma español, inglés y portugués (Motivation, BPNPE, Physical Education, Teacher support). Las bases de datos consultadas fueron Scielo, Dialnet, Redalyc y Google Scholar.

Se seleccionaron los principales estudios donde se reflejarán intervenciones y revisiones literarias sobre trabajos relacionados con el apoyo docente y las necesidades psicológicas básicas (Trigueros, 2020) específicamente en la educación física y el deporte a nivel internacional.

Los investigadores realizaron la búsqueda utilizando la lista de palabras clave para el presente análisis de los autores clásicos y contemporáneos.

Se efectuaron las siguientes instrucciones de selección para determinar si los artículos obtenidos en las bases de datos indexadas eran relevantes para el presente estudio: 1) lectura de los títulos, relevancia del tema, después de la revisión inicial se eliminaron los documentos no contemplados relevantes; 2) lectura de los resúmenes, si los resúmenes no facilitaban información relacionada con los criterios del tema o no estar disponibles en su totalidad, se excluyeron de la revisión; 3) lectura de los artículos en texto completo, si los artículos no cumplían con los criterios de inclusión, se excluyeron del estudio; 4) fiabilidad de los datos obtenidos y la calidad de los estudios seleccionados, apoyo significativo de las conclusiones que derivaron de los estudios. 


\section{Resultados}

A continuación, se presentan los principales resultados:

Grupo de investigaciones relacionadas con la motivación docente

Abraham Maslow, psicólogo estadounidense, nacido en el año 1908, desarrolló la teoría de la motivación en los seres humanos y establece una jerarquía para sustentar su teoría en las diversas necesidades humanas (Zapata \& Meza, 2007; Pomares 2008, citado en Pereira, 2009). La misma que se ha representado en forma de "La Pirámide de Maslow", según la cual, un ser humano tiende a satisfacer las necesidades primarias (más bajas en la pirámide), antes de buscar las de más alto nivel.

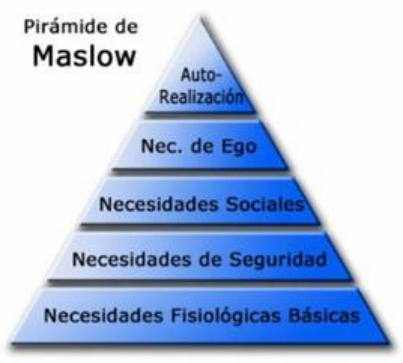

Figura 1. Pirámide de Maslow

Fuente: (Cosacov Eduardo, 2005)

De acuerdo con esta pirámide, algunos autores definen a la motivación: Sexton (1977: 162) menciona que es el proceso de estimular a un individuo para que realice una acción que satisfaga alguna de sus necesidades y alcance alguna meta deseada. De igual manera Armstrong (1991: 266) manifiesta que "son los recursos humanos fundamentales para mantener la cultura y los valores"; en el mismo sentido Robbins (1999: 17) menciona que "es un esfuerzo para alcanzar las metas deseadas, cuyo interés primordial son los valores para satisfacer las necesidades de los individuos", mientras que Stoner (1996) puntualiza que es "Una característica de la Psicología humana, e incluye los factores que ocasionan, canalizan y sostienen la conducta humana, lo que hace que las personas funcionen", a su vez Chiavenato (2000) la especifica como "el resultado de la interacción entre el individuo y la situación que lo rodea". Por su parte Mahillo (1996) manifiesta que la motivación es “el primer paso que nos lleva a la acción”. 
Las definiciones anteriores llevan a concluir que el individuo debe sentirse motivado para realizar cada una de sus acciones, según lo señala Gonçalves (2017) "la actividad física y la motivación se plantea como uno de los factores clave para fomentar estilos de vida saludables entre el alumnado", es decir, estar predispuesto a realizar diferentes actividades durante el proceso educativo, principalmente durante las actividades tendientes a satisfacer las necesidades emocionales, pues la motivación logra consecuencias positivas como: el esfuerzo, la concentración, la vitalidad, el desarrollo positivo o la intención de ser físicamente activo (Sánchez-Oliva et al., 2012).

Es así como el área de la Educación Física presenta grandes expectativas motivacionales, pues posee una variedad de actividades didácticas, donde se puede analizar la motivación y la enseñanza durante las prácticas escolarizadas. La educación física de ntro del currículo debe ser flexible, de acuerdo con las necesidades de los educandos, tanto en el aspecto físico, mental, y axiológico; apoyar las fortalezas, aprovechar la motivación, la cual tienen que ver con los aspectos emocionales (Bonilla, 2019), en definitiva, fortalecer las debilidades socioeducativas, donde cada debilidad propicie una fortaleza.

Tabla 1

Investigaciones relevantes relacionadas con el clima motivacional

\begin{tabular}{|c|c|c|c|c|}
\hline Autores & Título & Revista & Instrumentos Aplicados & $\begin{array}{c}\text { Relevancia/ } \\
\text { Número de } \\
\text { citas }\end{array}$ \\
\hline $\begin{array}{l}\text { González- } \\
\text { Cutre, D., } \\
\text { Sicilia, Á., \& } \\
\text { Fernández, A. } \\
\text { (2010). }\end{array}$ & $\begin{array}{l}\text { Hacia una mayor } \\
\text { comprensión de la } \\
\text { motivación en el } \\
\text { ejercicio físico: } \\
\text { medición de la } \\
\text { regulación } \\
\text { integrada en el } \\
\text { contexto español. }\end{array}$ & $\begin{array}{l}\text { Psicothema, 22(4), } \\
841-847 .\end{array}$ & $\begin{array}{l}\text { Cuestionario de la Regulación de } \\
\text { la Conducta en el Ejercicio. }\end{array}$ & 98 \\
\hline \multirow[t]{2}{*}{$\begin{array}{l}\text { Núñez Alonso, } \\
\text { J. L., Martín- } \\
\text { Albo, J., \& } \\
\text { Domínguez, E. } \\
\text { (2010). }\end{array}$} & $\begin{array}{l}\text { Análisis de las } \\
\text { propiedades } \\
\text { psicométricas de la } \\
\text { escala de } \\
\text { satisfacción con la } \\
\text { vida en sujetos } \\
\text { practicantes de } \\
\text { actividad física. }\end{array}$ & $\begin{array}{l}\text { Revista de } \\
\text { Psicología del } \\
\text { Deporte, 19(2), } \\
\text { 0291-304. }\end{array}$ & $\begin{array}{l}\text { Subescala Motivación Intrínseca } \\
\text { de la Perceived Locus of } \\
\text { Causality Scale (PLoC) } \\
\text { Subescala Autoconcepto Físico } \\
\text { General del Cuestionario de } \\
\text { Autoconcepto Físico (CAF) }\end{array}$ & 43 \\
\hline & & & $\begin{array}{l}\text { La Satisfaction with Life Scale } \\
\text { (swLs) }\end{array}$ & \\
\hline
\end{tabular}


Tabla 1 (continuación)

Investigaciones relevantes relacionadas con el clima motivacional

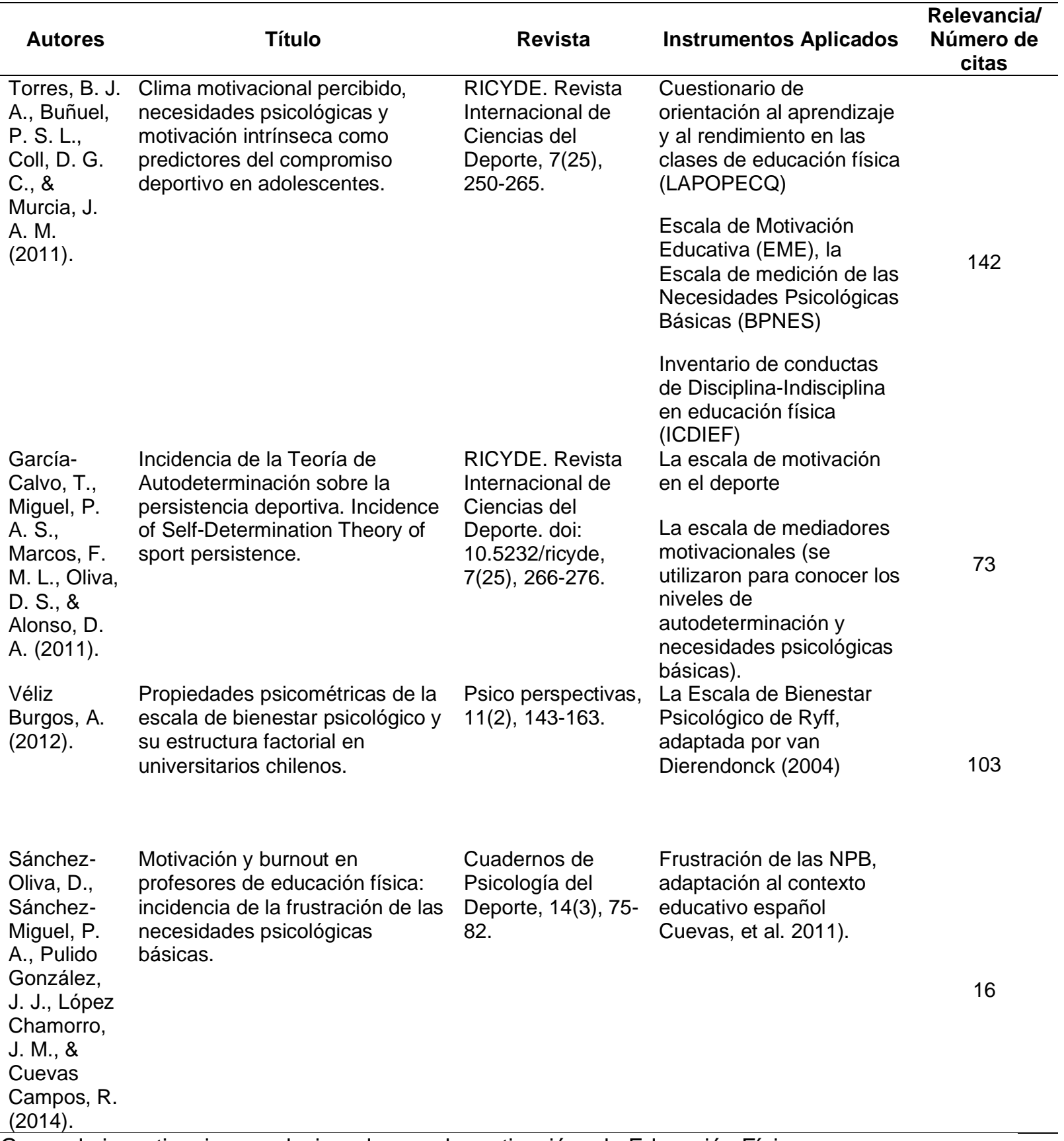

Grupo de investigaciones relacionadas con la motivación y la Educación Física

Dentro de la formación docente y la labor profesional en el ámbito educativo escolarizado se presentan dificultades, los cuales deben ser afrontados por los educadores durante el proceso 
de enseñanza de la Educación Física. Concordando con la opinión de Cimarro (2014) cuando considera "que como educadores estamos obligados a fomentar el desarrollo de planes de actividad física y salud adecuados, para ayudar a nuestros escolares a que perciban y valoren los beneficios de la actividad física regular, la correcta alimentación, el adecuado descanso y la higiene personal" citado en (Cáliz, 2016).

En los últimos años existe un interés creciente de fomentar la práctica de Educación Física activa y saludable, el incremento de la actividad física diaria, particularmente en los colegios, lo que contribuye a la salud de los niños y proporciona muchos valores educativos (Ahrabi-Fard, 2005); este paradigma lleva a pensar ¿cuál podría ser la estrategia a utilizarse? En tal sentido, se propone, indagar las Necesidades Psicológicas Básicas como una estrategia en la generación de una motivación autodeterminada (Deci \& Ryan, 1985,1991, 2000, 2002; Albo Martin 2005; Menéndez 2018).

Múltiples trabajos investigativos sustentan la Teoría de la Autodeterminación; cuyo instrumento conocido como Cuestionario de Apoyo a las Necesidades Psicológicas Básicas diseñado por (Sánchez-Oliva, Leo, Sánchez-Miguel, Amado \& García-Calvo 2015) desglosa tres factores motivacionales: autonomía, competencia y las relaciones sociales, propiciando los ambientes de aprendizaje en el área de la Educación Física. De acuerdo con esto se presenta la fundamentación teórica de soporte.

Tabla 2

Investigaciones relevantes relacionadas con la autodeterminación

\begin{tabular}{llllc}
\hline \multicolumn{1}{c}{ Autores } & \multicolumn{1}{c}{ Título } & \multicolumn{1}{c}{ Revista } & \multicolumn{1}{c}{ Instrumentos Aplicados } & $\begin{array}{c}\text { Relevancia } \\
\text { /Número de } \\
\text { citas }\end{array}$ \\
\hline MURCIA, J. & Complementando la & Revista & Metas sociales en educación física. & 87 \\
A. M., & teoría de la & Mexicana de & Se empleó la Social Goal Scale- & \\
PAÑOS, A. & autodeterminación con & Psicología, & Physical Education (SGS-PE) \\
H., \& COLL, & las metas sociales: un & $26(2), 213-$ & Escala de las Necesidades \\
D. G. C. & estudio sobre la diversión & 222. & Psicológicas Básicas en el Ejercicio \\
(2009). & en educación física. & & Escala del Locus Percibido de & \\
& & & Causalidad (PLOC) & \\
& & & Versión traducida al español por & \\
& & & García Calvo (2004) del Intrinsic & \\
& & & Motivation Inventory. & \\
\end{tabular}


Tabla 2 (continuación)

Investigaciones relevantes relacionadas con la autodeterminación

\begin{tabular}{|c|c|c|c|c|}
\hline Autores & Título & Revista & Instrumentos Aplicados & $\begin{array}{l}\text { Relevancia } \\
\text { /Número de } \\
\text { citas }\end{array}$ \\
\hline $\begin{array}{l}\text { Murcia, J. A. } \\
\text { M., Noguera, } \\
\text { F. C., Coll, } \\
\text { D. G. C., } \\
\text { Gimeno, E. } \\
\text { C., \& Pérez, } \\
\text { L. M. R. } \\
\text { (2009). }\end{array}$ & $\begin{array}{l}\text { Flow disposicional en } \\
\text { salvamento deportivo: } \\
\text { una aproximación } \\
\text { desde la teoría de la } \\
\text { autodeterminación. }\end{array}$ & $\begin{array}{l}\text { Revista de } \\
\text { Psicología del } \\
\text { Deporte, 18(1), 23- } \\
\text { 35. }\end{array}$ & $\begin{array}{l}\text { Perceived Motivational Climate in } \\
\text { Sport Questionnaire-2 (PMCSQ- } \\
\text { 2), the Motivational Mediators in } \\
\text { Sport Scale (EMMD), the Sport } \\
\text { Motivational Scale (SMS) and the } \\
\text { Dispositional Flow Scale (DFS) }\end{array}$ & 69 \\
\hline $\begin{array}{l}\text { Sánchez- } \\
\text { Oliva, D., } \\
\text { Viladrich, C., } \\
\text { Amado, D., } \\
\text { González- } \\
\text { Ponce, I., \& } \\
\text { García- } \\
\text { Calvo, T. } \\
\text { (2014). }\end{array}$ & $\begin{array}{l}\text { Predicción de los } \\
\text { Comportamientos } \\
\text { Positivos en Educación } \\
\text { Física: una perspectiva } \\
\text { desde la Teoría de la } \\
\text { Autodeterminación. }\end{array}$ & $\begin{array}{l}\text { Revista de psico } \\
\text { didáctica, 19(2), } \\
\text { 387-406. }\end{array}$ & $\begin{array}{l}\text { Cuestionario de Apoyo a las } \\
\text { Necesidades Psicológicas. } \\
\text { Cuestionario de Motivación en la } \\
\text { Educación Física (CMEF: } \\
\text { Sánchez-Oliva, Amado, Leo, } \\
\text { González-Ponce, y García-Calvo, } \\
\text { 2012). }\end{array}$ & 17 \\
\hline $\begin{array}{l}\text { Tomás, J. } \\
\text { M., \& } \\
\text { Gutiérrez, M. } \\
\text { (2019). }\end{array}$ & $\begin{array}{l}\text { Aportaciones de la } \\
\text { teoría de la } \\
\text { autodeterminación a la } \\
\text { predicción de la } \\
\text { satisfacción escolar en } \\
\text { estudiantes } \\
\text { universitarios. }\end{array}$ & $\begin{array}{l}\text { Revista de } \\
\text { Investigación } \\
\text { Educativa, 37(2), } \\
\text { 471-485. }\end{array}$ & $\begin{array}{l}\text { Percepción de Apoyo a la } \\
\text { Autonomía por los Profesores, } \\
\text { Satisfacción Necesidades de las } \\
\text { Psicológicas Básicas de los } \\
\text { Estudiantes, y Conectividad } \\
\text { Académica }\end{array}$ & 3 \\
\hline
\end{tabular}

Castuera (2015) en un estudio realizado en España con adolescentes estudiantes de segundo ciclo y primero de Bachillerato, analizó el Modelo Jerárquico de la motivación y los estudios de cambio para el ejercicio físico, empleando un diseño de investigación descriptivo transversal, que aplica el Cuestionario de Orientación al Aprendizaje y al Rendimiento en las Clases de Educación Física (LAPOPECQ) ${ }^{5}$, además de la Escala de Motivación Educativa EME) ${ }^{6}$, la Escala de medición de las Necesidades Psicológicas Básicas (BPNES) y el Cuestionario de Estados de Cambio para el ejercicio físico de medida continua (URICA-

${ }^{5}$ El Cuestionario de Orientación al Aprendizaje y al Rendimiento en las Clases de Educación Física (LAPOPECQ), originalmente de Papaioannou (1994) y traducido y validado al español (Cervelló\& Jiménez, 2001; Cervelló, Jiménez, Moya, \& Moreno, 2010), mide la percepción de los discentes acerca del clima motivacional que suelen experimentar en las clases de EF. Se compone de 27 ítems.

${ }^{6}$ La Escala de Motivación Educativa (EME) (Vallerand, Blais, Brière, \&Pelletier, 1989) analiza la motivación que poseen los discentes. Está traducida, validada al español por Núñez, Martín-Albo y Navarro (2005), con 28 ítems. 
E2) ${ }^{7}$. Los resultados más relevantes mostraron que la necesidad psicológica básica de competencia es la variable más relevante para adherirse a los estados de la práctica de ejercicio físico. La investigación presenta varios instrumentos utilizados para analizar la motivación, sin embargo, el que más llama la atención es el instrumento (BPNES) aplicando para medir las necesidades psicológicas básicas en el ejercicio. Se concluye, para el caso citado en España, el instrumento es una indagación original y relevante para el estudio realizado, con lo cual la escala (BPNES) se debe considerar como un instrumento referencial de partida para el caso del análisis del contexto ecuatoriano, pues a nivel del Ecuador no se han realizado investigaciones sobre el tema de la motivación en las prácticas de la educación física, considerando al instrumento como un apoyo para analizar la motivación y el comportamiento de los niños escolarizados.

Por otra parte, Rijo (2014) en su artículo "Necesidades Psicológicas Básicas en Educación Física según el género y el ciclo educativo del estudiante durante la escolaridad obligatoria" analizan los efectos del género y el ciclo educativo de los estudiantes sobre las necesidades psicológicas básicas en el área de Educación Física, tomando parte 684 alumnos de la asignatura de Educación Física (323 chicos y 361 chicas). Se utilizó la adaptación a la Educación Física de la escala de las necesidades psicológicas básicas en el ejercicio. Los autores consideran que las necesidades psicológicas básicas deben ser satisfechas para determinar una mayor motivación intrínseca en el área de la Educación Física. Esto lleva a plantear la importancia del estudio, pues el instrumento utilizado se considera como el adecuado para verificar la satisfacción personal de los alumnos de primaria para todo tipo de contextos, ya que, al estudiar la motivación durante los procesos pedagógicos de la Educación Física se puede indagar la conducta y motivación intrínseca, con lo cual se pretende alcanzar los verdaderos objetivos de la práctica física, conseguir una verdadera expresión de satisfacción socioeducativa y personal. Por otra parte, se han realizado estudios acerca de la motivación comparando el género chicos-chicas y su relación con el grado de diversión que experimentan los sujetos en la Educación Física. (Torres, B. J, Saénz-López, González-Cutre,

\footnotetext{
${ }^{7}$ Cuestionario de Estadios de Cambio para el Ejercicio Físico - Medida Continua El Cuestionario de Estadios de Cambio para el Ejercicio Físico - Medida Continua (URICA-E2), de Marcus et al. (1992), mide los estadios de cambio para el ejercicio físico. Moreno (2010) realizó la traducción y la validación al español. Se compone de 24 ítems.
} 
\& Moreno, 2011); citado en (Calvo, Oliva, \& Alonso, 2012). Los resultados muestran que, al existir mayor motivación, existe mayor grado de diversión, en este sentido se puede destacar la necesidad de fomentar el aspecto motivacional hacia la consecución de mejorar las sensaciones de diversión durante la práctica escolarizada de la Educación Física. En todo caso, el aspecto importante que debe hacer reflexionar a los docentes que imparten Educación Física en el ámbito educativo primario, convienen considerar el hecho de planificar los contenidos curriculares y basarlos en función de los intereses y las necesidades emocionales de sus educandos.

De igual manera, al investigar los efectos del género en la percepción de apoyo de las necesidades psicológicas básicas en la Educación Física Serrano (2015), llega a la conclusión que, los chicos presentan significativamente valores superiores en comparación con las chicas, especialmente dentro de la relación del apoyo a la competencia y de la autonomía, según el estudio se puede mencionar que los contextos educativos son muy diversos, especialmente el entorno educativo ecuatoriano, tanto en la situación geográfica, como de etnias y la situación socio cultural; por consiguiente, en el estudio para el contexto de investigaciones ecuatorianas se recomiendan dar mayor énfasis de apoyo al género femenino, tomando en cuenta el apoyo de las necesidades psicológicas básicas hacia las estudiantes del género femenino, sin embargo, no se puede descuidar a los estudiantes varones.

En base a la fundamentación científica presentada se puede mencionar que las investigaciones poseen originalidad, ya que los contextos escolares en América Latina presentan gran similitud con los contextos de las investigaciones descritas anteriormente, según lo cual, se puede dar respuesta a las interrogantes relacionadas con las necesidades Psicológicas básicas.

Al analizar las perspectivas teóricas más importante dentro de las teorías contemporáneas basadas en la autodeterminación, las Necesidades Psicológicas Básicas son muy utilizadas para explicar los procesos motivacionales en los contextos educativos en la Educación Física, contexto que ha adquirido gran auge en los últimos años (Ntoumanis y Standage, 2009); (Van den Berghe, Vansteenkiste, Cardon, Kirk, y Haerens, en prensa), la teoría sustenta ciertos factores educativo-sociales que promuevan diversos tipos de motivación en las personas Vallerand y Rousseau (2001), de igual manera apoya los tres tipos 
de necesidades psicológicas básicas que mantienen el bienestar personal: A).- competencia (capacidad de realizar acciones con la seguridad), B).- autonomía (capacidad para tomar decisión) y, C).- relación con los demás (considerarse importante). Con lo cual se puede referir, en la medida que estas necesidades sean fomentadas en los educandos se pueden desarrollar diversas formas de motivación más o menos autodeterminadas o volitivas (Moreno, González-Cutre, Martín-Albo, y Cervelló 2010).

Tabla 3

Investigaciones relevantes relacionadas con las necesidades psicológicas básicas en la educación física

\begin{tabular}{|c|c|c|c|c|}
\hline Autores & Título & Revista & Instrumentos Aplicados & $\begin{array}{c}\text { Relevancia } \\
\text { /Número de } \\
\text { citas }\end{array}$ \\
\hline $\begin{array}{l}\text { Castillo, E. } \\
\text { C., Almagro, } \\
\text { B. J., García, } \\
\text { C. C., \& } \\
\text { Buñuel, P. S. } \\
\text { L. (2015). }\end{array}$ & $\begin{array}{l}\text { Inteligencia } \\
\text { emocional y } \\
\text { motivación en } \\
\text { educación } \\
\text { física en } \\
\text { secundaria. }\end{array}$ & $\begin{array}{l}\text { Retos. Nuevas } \\
\text { Tendencias en } \\
\text { Educación } \\
\text { Física, Deporte y } \\
\text { Recreación, (27), } \\
\text { 8-13. }\end{array}$ & $\begin{array}{l}\text { La inteligencia emocional percibida (IEP) } \\
\text { El Cuestionario del Clima Motivacional } \\
\text { Percibido en el Deporte-2 (PMCSQ-2 } \\
\text { Perceived Locus of Causality Scale } \\
\text { Cuestionario de Responsabilidad } \\
\text { Personal y Social y la subescala de } \\
\text { Motivación Intrínseca de la Escala del } \\
\text { Locus Percibido de Casualidad } \\
\text { Basic Psychological Needsin Exercise } \\
\text { Scale (BPNES) }\end{array}$ & 55 \\
\hline $\begin{array}{l}\text { Moreno- } \\
\text { Murcia, J. A., } \\
\text { Gimeno, E. } \\
\text { C., } \\
\text { Carretero, C. } \\
\text { M., Lacárcel, } \\
\text { J. A. V., \& } \\
\text { Calvo, T. G. } \\
\text { (2012). }\end{array}$ & $\begin{array}{l}\text { Metas sociales, } \\
\text { necesidades } \\
\text { psicológicas } \\
\text { básicas y } \\
\text { motivación } \\
\text { intrínseca } \\
\text { como } \\
\text { predictores de } \\
\text { la percepción } \\
\text { del esfuerzo en } \\
\text { las clases de } \\
\text { educación } \\
\text { física. }\end{array}$ & $\begin{array}{l}\text { Revista de } \\
\text { psicología del } \\
\text { deporte, 21(2), } \\
215-221 .\end{array}$ & $\begin{array}{l}\text { Social Goal Scale-Physical Education } \\
\text { (SGS-PE) } \\
\text { Basic Psychological Needs in Exercise } \\
\text { Scale (BPNES) adapted to physical } \\
\text { education, Intrinsic motivation subscale } \\
\text { of thePerceived Locus of Causality } \\
\text { Scale (PLOC) } \\
\text { The effort subscale of the Intrinsic } \\
\text { Motivation Inventory (IMI). }\end{array}$ & 58 \\
\hline $\begin{array}{l}\text { Giménez, A. } \\
\text { M., Río, J. } \\
\text { F., \& } \\
\text { Estrada, J. } \\
\text { A. C. (2013). }\end{array}$ & $\begin{array}{l}\text { Climas } \\
\text { motivacionales, } \\
\text { necesidades, } \\
\text { motivación y } \\
\text { resultados en } \\
\text { Educación } \\
\text { Física. }\end{array}$ & $\begin{array}{l}\text { Aula abierta, } \\
41(1), 63-72 .\end{array}$ & $\begin{array}{l}\text { EI PMCSQ-2 sobre el clima motivacional } \\
\text { El BPNES relativo a las necesidades } \\
\text { psicológicas básicas } \\
\text { La escala motivación intrínseca del } \\
\text { PLOC }\end{array}$ & 66 \\
\hline
\end{tabular}


Tabla 3 (Continuación)

Investigaciones relevantes relacionadas con las necesidades psicológicas básicas en la educación física

\begin{tabular}{|c|c|c|c|c|}
\hline Autores & Título & Revista & Instrumentos Aplicados & $\begin{array}{l}\text { Relevancia } \\
\text { /Número de } \\
\text { citas }\end{array}$ \\
\hline $\begin{array}{l}\text { Oliva, D. } \\
\text { S., Marcos, } \\
\text { F. M. L., } \\
\text { Alonso, D. } \\
\text { A., } \\
\text { Campos, R. } \\
\text { C., \& } \\
\text { Calvo, T. } \\
\text { G. (2013). }\end{array}$ & $\begin{array}{l}\text { Desarrollo y } \\
\text { validación del } \\
\text { cuestionario } \\
\text { de apoyo a las } \\
\text { necesidades } \\
\text { psicológicas } \\
\text { básicas en } \\
\text { educación } \\
\text { física. }\end{array}$ & $\begin{array}{l}\text { Matricidal. } \\
\text { European } \\
\text { Journal of } \\
\text { Human } \\
\text { Movement, 30, } \\
53-71 .\end{array}$ & $\begin{array}{l}\text { Cuestionario de Apoyo a las } \\
\text { Necesidades Psicológicas Básicas } \\
\text { (CANPB) podría ser un instrumento } \\
\text { válido y fiable para analizar la } \\
\text { percepción de apoyo a la } \\
\text { autonomía, competencia y } \\
\text { relaciones sociales. }\end{array}$ & 50 \\
\hline $\begin{array}{l}\text { Patón, R. } \\
\text { N., } \\
\text { Fernández, } \\
\text { J. E. R., \& } \\
\text { Nemiña, R. } \\
\text { E. (2016). }\end{array}$ & $\begin{array}{l}\text { Análisis de la } \\
\text { satisfacción de } \\
\text { las } \\
\text { necesidades } \\
\text { psicológicas } \\
\text { básicas, } \\
\text { motivación y } \\
\text { disfrute en } \\
\text { Educación } \\
\text { Física en } \\
\text { Primaria. }\end{array}$ & $\begin{array}{l}\text { Sportis: Revista } \\
\text { Técnico- } \\
\text { Científica del } \\
\text { Deporte } \\
\text { Escolar, } \\
\text { Educación } \\
\text { Física y } \\
\text { Psicomotricidad, } \\
2(3), 439-455 .\end{array}$ & $\begin{array}{l}\text { la Escala de Medición de las } \\
\text { Necesidades Psicológicas Básicas } \\
\text { (BPNES) } \\
\text { Escala del Locus Percibido de } \\
\text { Causalidad en Educación Física } \\
\text { (PLOC Scale) } \\
\text { Escala de medida del disfrute en la } \\
\text { actividad física (PACES) }\end{array}$ & 21 \\
\hline $\begin{array}{l}\text { Navarro } \\
\text { Patón, R., } \\
\text { Basanta } \\
\text { Camiño, S., } \\
\text { \& Abelairas } \\
\text { Gómez, C. } \\
\text { (2017). }\end{array}$ & $\begin{array}{l}\text { Los juegos } \\
\text { cooperativos: } \\
\text { incidencia en } \\
\text { la motivación, } \\
\text { necesidades } \\
\text { psicológicas } \\
\text { básicas y } \\
\text { disfrute en } \\
\text { Educación } \\
\text { Primaria. }\end{array}$ & $\begin{array}{l}\text { Sportis, 3(3), } \\
589-604 .\end{array}$ & $\begin{array}{l}\text { Responsabilidad social versión } \\
\text { española del Personal and Social } \\
\text { Responsibility Questionnaire de Li, } \\
\text { Wright, Rukavina \& Pickering } \\
\text { (2008) validada por Escartí, } \\
\text { Gutiérrez \& Pascual (2011). } \\
\text { Necesidades Psicológicas básicas. } \\
\text { version española validada } \\
\text { (Menéndez \& Fernández-Río, en } \\
\text { prensa) del Basic Psychological } \\
\text { Needs in Physical Education Scale } \\
\text { (BPN-PE) deVlachopoulos, Katartzi } \\
\text { \& Kontou (2011). } \\
\text { Perceived Locus of Causality } \\
\text { (Goudas, Biddle \& Fox, 1994), } \\
\text { validada al español por Moreno } \\
\text { González-Cutre \& Chillón (2009). }\end{array}$ & 11 \\
\hline $\begin{array}{l}\text { Menéndez } \\
\text { Santurio, J. } \\
\text { I., \& } \\
\text { Fernández- } \\
\text { Río, J. } \\
\text { (2018). }\end{array}$ & $\begin{array}{l}\text { Versión } \\
\text { española de la } \\
\text { escala de } \\
\text { necesidades } \\
\text { psicológicas } \\
\text { básicas en } \\
\text { educación } \\
\text { física. }\end{array}$ & $\begin{array}{l}\text { Revista } \\
\text { Internacional de } \\
\text { Medicina y } \\
\text { Ciencias de la } \\
\text { Actividad Física } \\
\text { y del Deporte. }\end{array}$ & $\begin{array}{l}\text { Escala de las Necesidades } \\
\text { Psicológicas Básicas en el Ejercicio } \\
\text { (BPNES) }\end{array}$ & 19 \\
\hline
\end{tabular}


Tabla 3 (Continuación) Investigaciones relevantes relacionadas con las necesidades psicológicas básicas en la educación física

\begin{tabular}{|c|c|c|c|c|}
\hline Autores & Título & Revista & Instrumentos Aplicados & $\begin{array}{c}\text { Relevancia } \\
\text { /Número de } \\
\text { citas }\end{array}$ \\
\hline $\begin{array}{l}\text { Trigueros, } \\
\text { R., Aguilar- } \\
\text { Parra, J. } \\
\text { M., } \\
\text { González- } \\
\text { Santos, J., } \\
\text { \& Cangas, } \\
\text { A. J. } \\
\text { (2020). }\end{array}$ & $\begin{array}{l}\text { Validación y } \\
\text { adaptación de la } \\
\text { escala de control } \\
\text { psicológico del } \\
\text { profesor hacia las } \\
\text { clases de educación } \\
\text { física y su efecto } \\
\text { sobre las } \\
\text { frustraciones de las } \\
\text { necesidades } \\
\text { psicológicas básicas. }\end{array}$ & $\begin{array}{l}\text { Retos, } \\
37(37), \\
167-173 .\end{array}$ & $\begin{array}{l}\text { Controlling Teaching Scale (PCTs; } \\
\text { Soenens, et al., 2012). }\end{array}$ & 19 \\
\hline
\end{tabular}

\section{Conclusiones}

La enseñanza de la Educación Física es una de las disciplinas que se está desarrollando en América Latina. Una ciencia que adquiere importancia en el Ecuador, pues en los últimos años los procesos de inclusión e igualdad están fortaleciendo las prácticas educativas en la EF, ya que por medio de las actividades físicas los niños y jóvenes establecen formas de demostrar sus habilidades y potencialidades, por consiguiente, es necesario el apoyo docente hacia las necesidades psicológicas básicas en la educación física y el deporte.

La Educación Física escolar, constituye un proceso pedagógico muy importante dentro de la formación integral del ser humano, su enseñanza hoy en día es fundamental, razón por la cual a nivel mundial se realizan estudios para mejorar los procesos educativos, afianzando los valores a través de los procesos de motivación docente en base a programas y contenidos en el régimen escolarizado.

El desafío de los docentes de Educación Física será entonces, construir estrategias de enseñanza según las particularidades de los estudiantes, destacando el apoyo docente y la motivación en la construcción de aprendizajes significativos, destacando a la escala de las necesidades psicológicas básicas en la educación física como un instrumento de apoyo en la labor docente.

Finalmente, un aspecto que se debe tomar en cuenta dentro de la enseñanza de la asignatura de Educación Física es lo que señala Echeverría (2002) acerca de los procesos de 
motivación en la Educación Física para lograr una "influencia positiva en los valores cognoscitivos y afectivos, por ende, en la educación integral de los educandos".

\section{Referencias bibliográficas}

Ahrabi-Fard, I. \&. (2005). Promoción de una educación activa de la actividad física orientada a la salud en las clases de educación física. Cultura, Ciencia y Deporte, 1(3), 1(3), 163-170.

Aranda, A. F. (2013). El seminario colaborativo: una propuesta formativa para el profesorado de Educación Física. Contextos Educativos. Revista de Educación (5), 101-122.

Bonilla, J. A. M., Ricardo, Y. R., Fuente, C. D. C., \& López, X. C. M. (2019). La autoestima, la autonomía y el apoyo a las necesidades psicológicas básicas en personas con discapacidad visual. Ciencia Digital, 3(2.5), 120-133.

Cáliz, R. C. (2016). Influencia de la educación física en los hábitos saludables del alumnado de primer ciclo de educación secundaria en centros de las comarcas del sur de Córdoba. España: Tesis doctoral dirigida por María del Mar Cepero González (dir. tes.), Rosario Padial Ruz (codir. tes.) Universidad de Granada.

Castillo, E. C., Almagro, B. J., García, C. C., \& Buñuel, P. S. L. (2015). Inteligencia emocional y motivación en educación física en secundaria. Retos. Nuevas Tendencias en Educación Física, Deporte y Recreación, (27), 8-13.

Castuera, R. J. (2015). Castuera, R. J., Navarrete, B. M., Román, M. L., \& Rabaz, F. C. (2015). Motivación y estadios de cambio para el ejercicio físico en adolescentes. Revista Latinoamericana de Psicología, 47(3), 196-204.

Chiavenato, I. (2000). Administración de Recursos Humanos. México: Editorial McGraw Hill.

Cosacov Eduardo. (2005). Introducción a la psicología (quinta edición ed.). Córdova Argentina: Brujas. 
Echevarría L, J. A. (12 de Julio de 2002). http://www.efdeportes.com/ Revista Digital Buenos Aires - Año 8 - $\mathrm{N}^{\circ} 50$ - Julio de 2002. Obtenido de http://www.efdeportes.com/ Revista Digital - Buenos Aires - Año 8 - N 50 - Julio de 2002: http://www.efdeportes.com/efd50/claseef.htm

García-Calvo, T., Miguel, P. A. S., Marcos, F. M. L., Oliva, D. S., \& Alonso, D. A. (2011). Incidencia de la Teoría de Autodeterminación sobre la persistencia deportiva. (Incidence of Self-Determination Theory of sport persistence). RICYDE. Revista Internacional de Ciencias del Deporte. doi: 10.5232/ricyde, 7(25), 266-276.

Giménez, A. M., Río, J. F., \& Estrada, J. A. C. (2013). Climas motivacionales, necesidades, motivación y resultados en Educación Física. Aula abierta, 41(1), 63-72.

Gonçalves, I. C. (2017). Tipos de motivación para participar en las clases de educación física en la etapa de enseñanza secundaria obligatoria (ESO). Acción Motriz, 18, 37-46.

González-Cutre, D., Sicilia, Á., \& Fernández, A. (2010). Hacia una mayor comprensión de la motivación en el ejercicio físico: medición de la regulación integrada en el contexto español. Psicothema, 22(4), 841-847.

Gutiérrez, M. \&. (2016). Clima motivacional, razones para la disciplina y comportamiento en Educación Física/Motivational climate, reasons for discipline and behavior in physical education. Revista Internacional de Medicina y Ciencias de la Actividad Física y del Deporte, (46)., 1-17.

Menéndez Santurio, J. I., \& Fernández-Río, J. (2018). Versión española de la escala de necesidades psicológicas básicas en educación física. Revista Internacional de Medicina y Ciencias de la Actividad Física y del Deporte.

Moreno, B. J. (2011). Análisis de la percepción del clima motivacional, necesidades psicológicas básicas, motivación autodeterminada y conductas de disciplina de estudiantes adolescentes en las clases de educación física. European Journal of Human Movement, 1(26), 1-24. 
Moreno-Murcia, J. A., Gimeno, E. C., Carretero, C. M., Lacárcel, J. A. V., \& Calvo, T. G. (2012). Metas sociales, necesidades psicológicas básicas y motivación intrínseca como predictores de la percepción del esfuerzo en las clases de educación física. Revista de psicología del deporte, 21(2), 215-221.

Moreno-Murcia, J. A., Marzo, J. C., Martínez-Galindo, C., \& Marín, L. C. (2011). Validación de la Escala de "Satisfacción de las Necesidades Psicológicas Básicas" y del Cuestionario de la "Regulación Conductual en el Deporte" al contexto español. (Validation of Psychological Need Satisfaction in Exercise Scale and the Behavioural Regulation in Sport Questionnaire to the Spanish context). RICYDE. Revista Internacional de Ciencias del Deporte. doi: 10.5232/ricyde, 7(26), 355-369.

MURCIA, J. A. M., PAÑOS, A. H., \& COLL, D. G. C. (2009). Complementando la teoría de la autodeterminación con las metas sociales: un estudio sobre la diversión en educación física. Revista Mexicana de Psicología, 26(2), 213-222.

Murcia, J. A. M., Noguera, F. C., Coll, D. G. C., Gimeno, E. C., \& Pérez, L. M. R. (2009). Flow disposicional en salvamento deportivo: una aproximación desde la teoría de la autodeterminación. Revista de Psicología del Deporte, 18(1), 23-35.

Navarro Patón, R., Basanta Camiño, S., \& Abelairas Gómez, C. (2017). Los juegos cooperativos: incidencia en la motivación, necesidades psicológicas básicas y disfrute en Educación Primaria. Sportis, 3(3), 589-604.

Núñez Alonso, J. L., Martín-Albo, J., \& Domínguez, E. (2010). Análisis de las propiedades psicométricas de la escala de satisfacción con la vida en sujetos practicantes de actividad física. Revista de Psicología del Deporte, 19(2), 0291-304.

Oliva, D. S., Marcos, F. M. L., Alonso, D. A., Campos, R. C., \& Calvo, T. G. (2013). Desarrollo y validación del cuestionario de apoyo a las necesidades psicológicas básicas en educación física. Motricidad. European Journal of Human Movement, 30, 53-71. 
Organización Mundial de la Salud. (21 de Diciembre de 2016). 10 datos sobre las enfermedades no transmisibles. Obtenido de 10 datos sobre las enfermedades no transmisibles: http://www.who.int/features/factfiles/noncommunicable_diseases/es/

Paiderex. (12 de 03 de 2011). http://revista.academiamaestre.es/2011/03/la-salud-en-el-areade-educacion-fisica/. Recuperado el 26 de 02 de 2015, de http://revista.academiamaestre.es/2011/03/la-salud-en-el-area-de-educacion-fisica/: http://revista.academiamaestre.es/2011/03/la-salud-en-el-area-de-educacion-fisica/

Patón, R. N., Fernández, J. E. R., \& Nemiña, R. E. (2016). Análisis de la satisfacción de las necesidades psicológicas básicas, motivación y disfrute en Educación Física en Primaria. Sportis: Revista Técnico-Científica del Deporte Escolar, Educación Física y Psicomotricidad, 2(3), 439-455.

Pereira. (2009). Motivación: perspectivas teóricas y algunas consideraciones de su importancia en el ámbito educativo. Revista Educación, 33(2), 153-170.

Rijo, A. G. (2014). Necesidades psicológicas básicas en Educación Física según el género y el curso del estudiante. Revista de Investigación Educativa, 1(32), 159-167.

Sánchez-Oliva, D. M.-G.-C. (2015). Análisis de los perfiles motivacionales y su relación con los comportamientos adaptativos en las clases de educación física. Revista Latinoamericana de Psicología, 3(47), 156-166.

Sánchez-Oliva, D., Marcos, F. M. L., Amado, D., Alonso, I. G. P., \& García-Calvo, T. (2012). Desarrollo de un cuestionario para valorar la motivación en educación física. Revista Iberoamericana de Psicología del Ejercicio y el Deporte VOL. VII No 2, 7(2), 226.

Sánchez-Oliva, D., Sánchez-Miguel, P. A., Pulido González, J. J., López Chamorro, J. M., \& Cuevas Campos, R. (2014). Motivación y burnout en profesores de educación física: incidencia de la frustración de las necesidades psicológicas básicas. Cuadernos de Psicología del Deporte, 14(3), 75-82. 
Sánchez-Oliva, D., Viladrich, C., Amado, D., González-Ponce, I., \& García-Calvo, T. (2014). Predicción de los Comportamientos Positivos en Educación Física: una perspectiva desde la Teoría de la Autodeterminación. Revista de psicodidáctica, 19(2), 387-406.

Serrano, J. S. (2015). Efectos del género en la percepción de apoyo de las necesidades psicológicas básicas en educación física. EmásF: revista digital de educación física, 1(34), 114-124.

Tomás, J. M., \& Gutiérrez, M. (2019). Aportaciones de la teoría de la autodeterminación a la predicción de la satisfacción escolar en estudiantes universitarios. Revista de Investigación Educativa, 37(2), 471-485.

Torres, B. J. A., Buñuel, P. S. L., Coll, D. G. C., \& Murcia, J. A. M. (2011). Clima motivacional percibido, necesidades psicológicas y motivación intrínseca como predictores del compromiso deportivo en adolescentes. RICYDE. Revista Internacional de Ciencias del Deporte, 7(25), 250-265.

Trigueros, R., Aguilar-Parra, J. M., González-Santos, J., \& Cangas, A. J. (2020). Validación y adaptación de la escala de control psicológico del profesor hacia las clases de educación física y su efecto sobre las frustraciones de las necesidades psicológicas básicas. Retos, 37(37), 167-173.

Velázquez, Walda. (14 de Noviembre de 2011). Clase de educación física escuela especializada en bellas artes de Humacao. obtenido de clase de educación física escuela especializada en bellas artes de Humacao: http://edufisicaebba.blogspot.com/

Véliz Burgos, A. (2012). Propiedades psicométricas de la escala de bienestar psicológico y su estructura factorial en universitarios chilenos. Psicoperspectivas, 11(2), 143-163.

Zapata, \& Meza. (2007). Análisis desde la teoría motivacional de las causas de ingreso y deserción del estudiante universitario. Scientia Et Technica, 1(37).

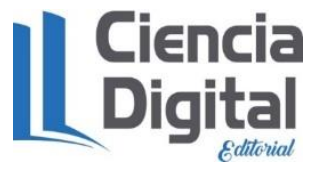




\section{PARA CITAR EL ARTÍCULO INDEXADO}

Mocha Bonilla, J. A., Comas Benítez, M., \& Galak, E. L. (2020). Apoyo motivacional docente y necesidades psicológicas básicas en la Educación Física: una revisión sistemática. Ciencia Digital, 4(1.1.), 5-20. https://doi.org/10.33262/cienciadigital.v4i1.1.1160

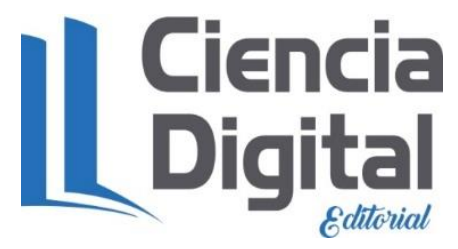

El artículo que se publica es de exclusiva responsabilidad de los autores y no necesariamente reflejan el pensamiento de la Revista Ciencia Digital.

El artículo queda en propiedad de la revista y, por tanto, su publicación parcial y/o total en otro medio tiene que ser autorizado por el director de la Revista Ciencia Digital.
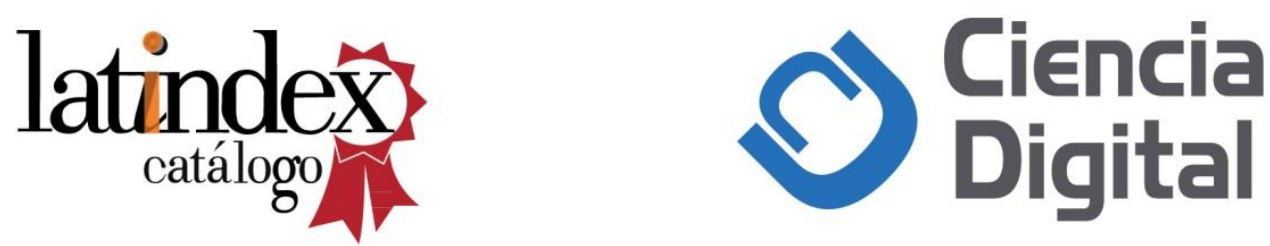